\title{
Helicoidal ordering in iron perovskites
}

\author{
Maxim Mostovoy \\ Max-Planck-Institut für Festkörperforschung, \\ Heisenbergstrasse 1, D-70569 Stuttgart, Germany
}

(Dated: August 31, 2004)

\begin{abstract}
We consider magnetic ordering in materials with negative charge transfer energy, such as iron perovskite oxides. We show that for a large weight of oxygen holes in conduction bands, the double exchange mechanism favors a helicoidal rather than ferromagnetic spin ordering both in metals, e.g. $\mathrm{SrFeO}_{3}$ and insulators with a small gap, e.g. $\mathrm{CaFeO}_{3}$. We discuss the magnetic excitation spectrum and effects of pressure on magnetic ordering in these materials.

PACS numbers: 75.10.-b,71.30.+h,75.50.Ee,75.30.-m
\end{abstract}

The interaction of itinerant electrons with localized spins couples transport to magnetism and gives rise to many fascinating phenomena, e.g., the heavy fermion and Kondo insulator behavior. In manganese perovskites the strong Hund's rule coupling between the $e_{g}$ and $t_{2 g}$ electrons results in the colossal magnetoresistance near the metal-insulator transition [1]. The transport and magnetic properties of manganites are furthermore strongly influenced by the instability of the partially filled $e_{g}$ orbitals towards an orbital ordering.

One would expect to find a similar behavior in other transition metal (TM) oxides with partially filled $e_{g}$ and $t_{2 g}$ levels. However, the iron perovskites $\mathrm{SrFeO}_{3}$ and $\mathrm{CaFeO}_{3}$ have very different properties. Although the tetravalent iron has the same $t_{2 g}^{3} e_{g}^{1}$ electronic configuration as the $\mathrm{Mn}^{3+}$ ion in $\mathrm{LaMnO}_{3}$, the ferrates, which lie closer to the borderline separating metals and insulators than manganites, exhibit no Jahn-Teller instability: $\mathrm{SrFeO}_{3}$ is a cubic metal down to lowest temperatures [2], while $\mathrm{CaFeO}_{3}$ is a charge-ordered insulator below $290 \mathrm{~K}$ [3]. Both the metallic and insulating ferrates show the helicoidal magnetic (HM) ordering with a small helix wave vector $\mathbf{Q}$ along the body diagonal, which sets in at $134 \mathrm{~K}$ in $\mathrm{SrFeO}_{3}$ and at $115 \mathrm{~K}$ in $\mathrm{CaFeO}_{3}$ [4, 5, 6]. A further evidence for the decoupling of transport and magnetism in these materials is the absence of a resistivity anomaly at Néel temperature in $\mathrm{SrFeO}_{3}$ [7].

A possible explanation of the suppression of orbital ordering in $\mathrm{Fe}^{4+}$ oxides came from photoemission experiments [8], which showed that $\mathrm{SrFeO}_{3}$ and $\mathrm{CaFeO}_{3}$ have a large negative charge transfer energy $\Delta \sim-3 \mathrm{eV}$ (i.e., the energy necessary to transfer an electron from an oxygen to a TM ion). For large negative $\Delta$, the conduction bands are formed by the strongly hybridized iron $e_{g}$ and oxygen $p_{\sigma}$ orbitals and the nominal $d^{4}$ state of the iron ions has a high weight of the $d^{5}$ state with holes on oxygen sites. Since the high-spin $d^{5}$ configuration is non-degenerate, the orbital ordering is suppressed. Furthermore, for the half filled $d$-orbital, the charge fluctuations shift from TM sites to Fe-O and O-O bonds, reducing the Coulomb energy cost of the charge ordering.

In this paper we discuss the origin of the helicoidal magnetism in ferrates. Since magnetism and transport in these materials seem to be largely decoupled, such an ordering cannot result from the spin-densitywave (SDW) or any other Fermi surface instability. The Dzyaloshinskii-Moria interaction [9], responsible for the $\mathrm{HM}$ ordering in, e.g., $\mathrm{MnSi}$, is forbidden by symmetry in the cubic $\mathrm{SrFeO}_{3}$. A non-collinear spin ordering can be a result of the competition between the ferromagnetic (FM) double exchange (DE) 10, 11] and the antiferromagnetic (AFM) superexchange (SE) between the spins of the $t_{2 g}$ electrons, as was discussed by de Gennes in Ref. 12]. However, even if we leave aside problems with the phase separation in DE systems [13], the HM state resulting from such a competition, only occurs at low concentrations of charge carriers, for which the kinetic energy of the $e_{g}$ electrons is comparable with the small superexchange energy of the $t_{2 g}$ electrons (the same holds for the spiral state in the $t J$-model of doped cuprates [14]). In particular, a non-collinear magnetic ordering in $\mathrm{La}_{1-x} \mathrm{Sr}_{x} \mathrm{MnO}_{3}$ was reported for $x=0.06-0.09$ [15. Furthermore, the delicate balance between the DE and $\mathrm{SE}$, necessary to stabilize the HM state, is incompatible with the insensitivity of magnetism to transport properties (and vice versa) in $\mathrm{SrFeO}_{3}$ and $\mathrm{CaFeO}_{3}$.

Here we establish a relation between the helicoidal ordering and suppression of orbital ordering in ferrates. We show that when the oxygen orbitals are included in the DE model and the density of oxygen holes is high, the kinetic energy of itinerant electrons is minimized for a helicoidal spin ordering. This result holds both for metals and small gap insulators.

The model: We consider an extended version of the DE model, which in addition to itinerant $e_{g}$ electrons and spins of localized $t_{2 g}$ electrons, includes the oxygen $\sigma$-orbitals that are strongly hybridized with the TM $e_{g^{-}}$ orbitals. It is convenient to describe states of the model in terms of holes that can occupy both oxygen and TM sites. By the holes on iron sites we mean the $e_{g}$-holes in the high-spin electronic $d^{5}$-configuration (the total spin $S=5 / 2$ ). For infinite Hund's rule coupling, the spin of the $e_{g}$ hole on the site $j$ is antiparallel to the local spin $\mathbf{S}_{j}$. Therefore, these holes can be described by the 
spinless operators $d_{j \alpha}$, where the index $\alpha=1,2$ denotes, respectively, the $3 z^{2}-r^{2}$ and $x^{2}-y^{2}$ orbitals. The holes on oxygen sites can have both spin projections: $p_{j \pm b / 2}=$ $\left(\begin{array}{c}p_{j \pm b / 2 \uparrow} \\ p_{j \pm b / 2 \downarrow}\end{array}\right)$, where $j \pm b / 2(b=x, y, z)$ are the 6 oxygen sites from the octahedron centered at the TM site $j$.

The Hamiltonian of the DE $d p$-model has the form

$$
\begin{aligned}
H_{d p} & =\sum_{j \alpha b} t_{\alpha b}\left(d_{j \alpha}^{\dagger} u_{j}^{\dagger} P_{j b}+P_{j b}^{\dagger} u_{j} d_{j \alpha}\right)+t_{p p} \sum_{j, b \neq c} P_{j b}^{\dagger} P_{j c} \\
& +\Delta \sum_{j b} p_{j+b / 2}^{\dagger} p_{j+b / 2}
\end{aligned}
$$

where the $d p-$ and $p p$-hopping amplitudes are expressed through the Slatter-Koster parameters by $t_{1}=$ $(p d \sigma)\left(-\frac{1}{2},-\frac{1}{2}, 1\right), t_{2}=(p d \sigma)\left(\frac{\sqrt{3}}{2},-\frac{\sqrt{3}}{2}, 0\right)$, and $t_{p p}=$ $\frac{1}{2}(p p \sigma)-\frac{1}{2}(p p \pi)$. Since for infinite Hund's rule coupling the hopping on the TM site $j$ is only possible for holes with the spin antiparallel to the local spin $\mathbf{S}_{j}=S\left(\sin \theta_{j} \sin \phi_{j}, \cos \theta_{j} \sin \phi_{j}, \cos \theta_{j}\right)$, the oxygen hole operator $P_{j b}=p_{j+b / 2}+p_{j-b / 2}$ is projected on the spinor

$$
u_{j}=\left(\begin{array}{c}
-\sin \frac{\theta_{j}}{2} e^{i \phi_{j}} \\
\cos \frac{\theta_{j}}{2}
\end{array}\right) .
$$

For HM state with the wave vector $\mathbf{Q}$ and spin rotation axis $\mathbf{e}_{\mathbf{3}}$ the local spin on the site $j$

$$
\mathbf{S}_{j}=S\left(\mathbf{e}_{1} \cos \mathbf{Q} \mathbf{x}_{j}+\mathbf{e}_{2} \sin \mathbf{Q} \mathbf{x}_{j}\right),
$$

where the unit vectors $\mathbf{e}_{1}, \mathbf{e}_{2}$, and $\mathbf{e}_{3}$ form an orthogonal basis. Due to the invariance of the tight-binding Hamiltonian (11) under an arbitrary rotation of the spin axes, the energy of the HM state is independent of $\mathbf{e}_{3}$. In $\mathrm{Sr}(\mathrm{Ca}) \mathrm{FeO}_{3}$ the spin rotation axis is parallel to the helix wave vector [5, 6] due to the anisotropy of spin interactions, which we neglect here. For $\mathbf{e}_{3}=\hat{x}$ and $\mathbf{e}_{\mathbf{1}}=\hat{z}$, we have $u_{j}=e^{\frac{i}{2} \sigma_{x}\left(\mathbf{Q x _ { j }}\right)}\left(\begin{array}{l}0 \\ 1\end{array}\right)$.

To obtain the hole energies for the HM state, the transformation to the momentum space has to be combined with the spin rotation:

$$
p_{j \pm b / 2}=\frac{1}{\sqrt{N}} \sum_{\mathbf{k}} e^{\left(\mathbf{k}+\frac{1}{2} \sigma_{x} \mathbf{Q}\right) \mathbf{x}_{j \pm b / 2}} p_{\mathbf{k} b}
$$

where $N$ is the number of Fe ions. The Hamiltonian (11) then reads

$$
\begin{aligned}
H & =\sum_{\mathbf{k} \alpha b} t_{\alpha b}\left(d_{\mathbf{k} \alpha}^{\dagger} P_{\mathbf{k} b \downarrow}+P_{\mathbf{k} b \downarrow}^{\dagger} d_{\mathbf{k} \alpha}\right)+t_{p p} \sum_{\mathbf{k}, b \neq c} P_{\mathbf{k} b}^{\dagger} P_{\mathbf{k} c} \\
& +\Delta \sum_{\mathbf{k} b} p_{\mathbf{k} b}^{\dagger} p_{\mathbf{k} b},
\end{aligned}
$$

where

$$
P_{\mathbf{k} b \sigma}=2\left(\cos \frac{Q_{b}}{4} \cos \frac{k_{b}}{2} p_{\mathbf{k} b \sigma}-\sin \frac{Q_{b}}{4} \sin \frac{k_{b}}{2} p_{\mathbf{k} b,-\sigma}\right)
$$

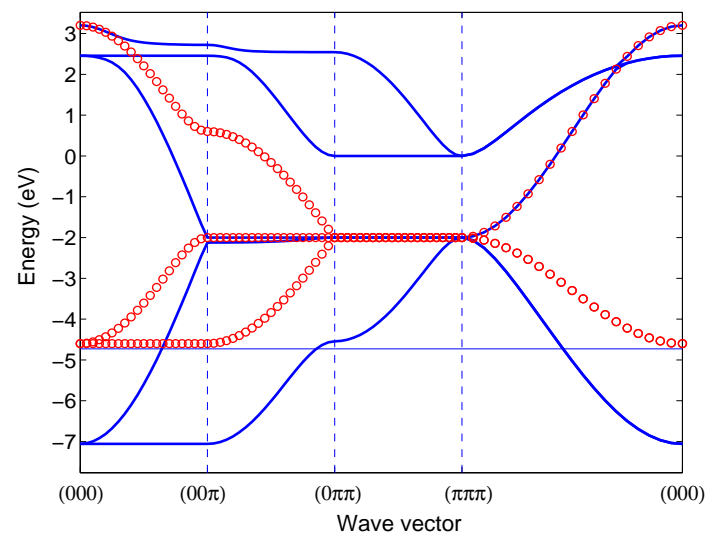

FIG. 1: The mixed $d p \downarrow$-hole bands (solid lines) and oxygen $p$ 个-hole bands (circles) for ferromagnetically ordered local spins, $(p d \sigma)=1.7 \mathrm{eV}, t_{p p}=0.65 \mathrm{eV}$, and $\Delta=-2 \mathrm{eV}$. The thin horizontal line indicates the Fermi level for $1 e_{g}$-hole/Fe.

(the lattice constant equals 1 ).

The hole bands for the FM state are shown in Fig. [1 For local spins oriented up, the spin-down bands (solid lines) are formed by the mixed $d-$ and $p$-hole states, while the spin-up bands (circles) are purely oxygen-hole bands. To a good approximation the states forming the two lowest bands are Bloch superpositions of the ZhangRice states of holes on metal-oxygen octahedra [16] with the symmetry of the $3 z^{2}-r^{2}$ and $x^{2}-y^{2}$ orbitals. These are the two bands of the effective $d d$-model used to describe colossal magnetoresistance manganites [13]. The Hilbert space of this model is reduced to the subspace of the TM orbitals. Even for ferrates with a large negative $\Delta$, the Fermi sea is either completely or predominantly filled by the states from these two bands (in Fig. 1 the Fermi energy $\varepsilon_{F}$ is indicated by the thin horizontal line).

Yet, for negative $\Delta$ the $d d$-model may fail to describe the magnetic ground state. The reason is the high density of low energy spin-flip excitations, created by promoting a hole from the spin-down Fermi sea to one of the two lowest oxygen spin-up bands, which at the $\Gamma$-point also have the symmetries of the $3 z^{2}-r^{2}$ and $x^{2}-y^{2}$ orbitals (see Fig. 10. In the HM state these bands become mixed with the two lowest $d p$-bands, which lowers the energies of the occupied states. There is also an energy loss due to the narrowing of bands caused by the relative rotation of local spins (the usual DE mechanism). When the bottom of the oxygen spin-up bands (at $\varepsilon=\Delta-4 t_{p p}$ ) is close to $\varepsilon_{F}$, the energy gain exceeds the energy loss, which makes the FM state unstable and stabilizes the HM state.

Although both the instability of the FM state towards the HM ordering and SDW instabilities are driven by the lowering of the energy of occupied states, the former does not require a nested Fermi surface and, in general, does not open a gap, since the crossing of the spin-up and spin-down bands at the Fermi surface only occurs at isolated points. While the energy driving an SDW 

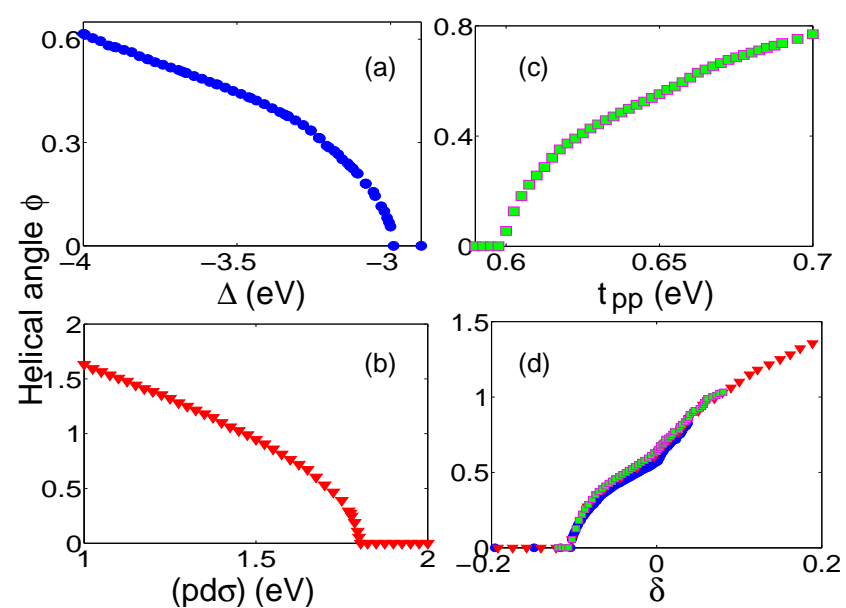

FIG. 2: The helical angle $\phi$ (in radians) between the spins in neighboring [111] layers plotted versus: (a) $\Delta$, for $(p d \sigma)=$ $1.8 \mathrm{eV}$ and $t_{p p}=0.6 \mathrm{eV}$; (b) the hopping amplitude $(p d \sigma)$, for $\Delta=-3 \mathrm{eV}$ and $t_{p p}=0.6 \mathrm{eV}$; (c) $t_{p p}$, for $(p d \sigma)=1.8 \mathrm{eV}$ and $\Delta=-3 \mathrm{eV}$. These three curves are re-plotted versus the separation between the Fermi energy and the bottom of the oxygen band in the FM state $\delta=\left(\varepsilon_{F}-\Delta+4 t_{p p}\right) /(p d \sigma)$ in panel (d).

transition is gained close to $\varepsilon_{F}$, in our case the energy gain is distributed over the whole Fermi sea - it is a Fermi sea rather than a Fermi surface instability.

The transition from the FM to HM ground state, induced by varying $\Delta,(p d \sigma)$, and $t_{p p}$, is shown Fig. 2 where we plot the optimal angle $\phi$ between the spins in neighboring [111] layers $(\phi=0$ corresponds to the FM state). In general, the helical angle $\phi$ grows with the weight of oxygen holes in the ground state, which can be achieved by decreasing $\Delta$ (see Fig. $2 a$ ) or the width of the hybridized $d p$-bands (see Fig. 20 ), or by increasing the width of the oxygen bands (see Fig. 2r). To a good approximation the value of $\phi$ is controlled by a single parameter $\delta=\left(\varepsilon_{F}-\Delta+t_{p p}\right) /(p d \sigma)$, describing the separation between the Fermi energy and the bottom of the oxygen spin-up band in the FM state. When the data shown in Figs. 2] a, b, and c are re-plotted versus $\delta$, they nearly fall on a single curve (see Fig. 21 d). The FM state becomes unstable for small negative $\delta$, when the bottom of the oxygen band is slightly above the Fermi energy. The phase diagram of the $d p$-model Eq. (1) is plotted in Fig. 31. For large $-\frac{\Delta}{(p d \sigma)}$ and $\frac{t_{p p}}{(p d \sigma)}$, the HM state has a lower energy than the FM one. The ratio of $\Delta$ and $(p d \sigma)$ can be decreased by pressure, which widens the conduction bands. Experimentally the switching from HM to FM state was observed in $\mathrm{SrFeO}_{3}$ at $\sim 13 \mathrm{GPa}$ [17.

Effect of superexchange: The AFM superexchange,

$$
H_{S E}=J \sum_{i b}\left(\mathbf{S}_{i} \mathbf{S}_{i+b}\right), \quad J>0
$$

between the spins of the $t_{2 g}$ electrons stabilizes a $\mathrm{HM}$
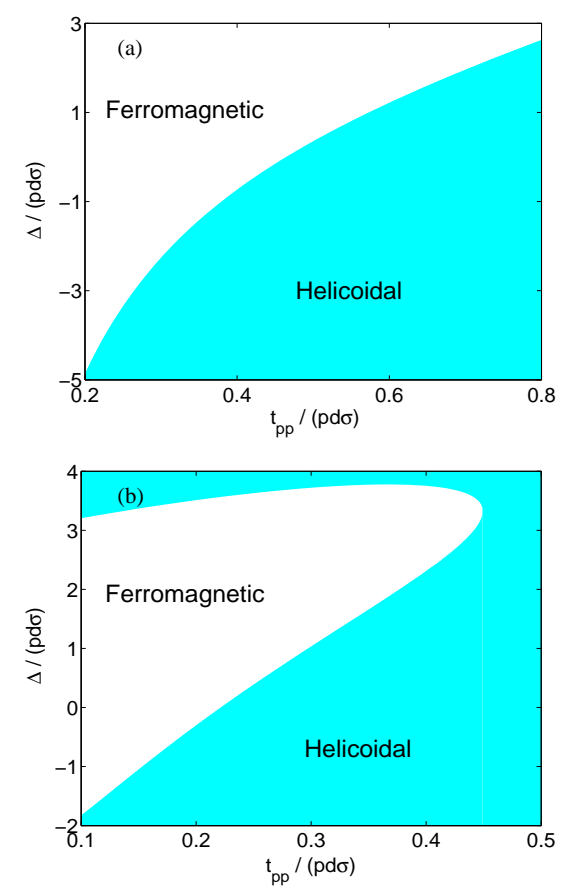

FIG. 3: The phase diagram of the $d p$-model (a) and the $d p$-model with the SE interaction (b), for $\frac{3 J S^{2}}{(p d \sigma)}=0.07$.

state also at large $\frac{\Delta}{(p d \sigma)}$ (see Fig. [3b), since for $\Delta \gg$ $(p d \sigma), H_{d p}+H_{S E}$ reduces to the DE model considered by de Gennes [12]. We believe, however, that the HM state found in $\mathrm{SrFeO}_{3}$ lies below the FM region rather than above it. The 'upper' helicoidal state only appears for very large positive $\Delta$. Furthermore, $\frac{3 J S^{2}}{(p d \sigma)}=0.07$ used to obtain Fig. [3], is too large for $\mathrm{SrFeO}_{3}$. The applicability of the de Gennes mechanism to $\mathrm{SrFeO}_{3}$ would require a strong reduction of hopping amplitudes, e.g., due to polaronic or strong correlation effects.

Magnetic ordering in insulators: Since the instability of the FM state towards HM ordering, is a Fermi sea rather than a Fermi surface instability, it can also occur in insulators, such as $\mathrm{CaFeO}_{3}$ below $T_{C O}=290 \mathrm{~K}$. Here we assume that the charge ordering in this material occurs due to a strong electron-lattice coupling, which for one hole/Fe gives rise to a breathing-type lattice distortion (an alternation of large and small oxygen octahedra), observed below $T_{C O}[6]$. Figure 4 shows the dependence of the gap in the hole spectrum (squares) due to the modulation of the hopping amplitudes in the ordered state: $t_{\alpha b}(i)=\left(1+\sigma_{i} \eta\right) t_{\alpha b}$ and $t_{p p}(i)=\left(1+\sigma_{i} \eta\right) t_{p p}$, where $\sigma_{i}= \pm 1$ for $i \in A / B$ sublattice of the cubic lattice. Since the Fermi surface is not nested, the gap only opens above some critical value of the modulation amplitude $\eta$ (a finite electron-phonon coupling constant is necessary to stabilize the charge ordered state). The helical angle $\phi$ (circles) decreases very slowly with $\eta$ and even the $1 \mathrm{eV}$ gap does not have a strong effect on the wave vector of 


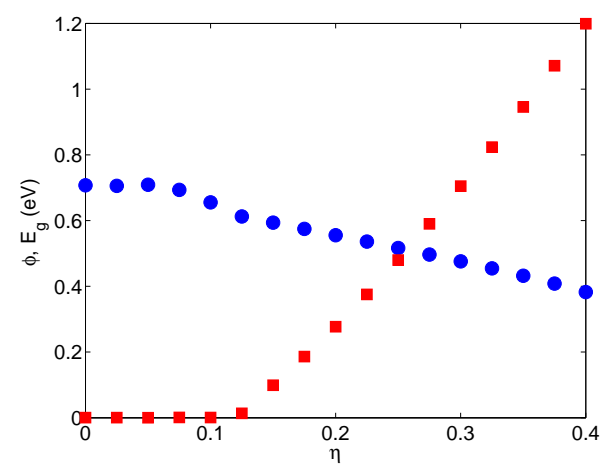

FIG. 4: The helical angle $\phi$ (circles), measured in radians, and the gap $E_{g}$ (squares), measured in $\mathrm{eV}$, versus the modulation amplitude $\eta$ for $(p d \sigma)=1.7 \mathrm{eV}, t_{p p}=0.65 \mathrm{eV}$, and $\Delta=-3 \mathrm{eV}$.

the HM ordering.

Magnon dispersion: The magnon spectrum for a HM state, calculated in the leading $1 / S$ approximation, is shown in Fig. 5 (details of this calculation will be published elsewhere). The magnon frequency vanishes both at $\mathbf{q}=0$ and $\mathbf{q}=\mathbf{Q}$, corresponding to the two Goldstone modes: the translation of the incommensurate helix along $\mathbf{Q}$ and rotation of the helical axis $\mathbf{e}_{3}$, respectively. Although the HM state is stable, the magnon spectrum for $\mathbf{q}$ varying between 0 and $\mathbf{Q}$ is extremely soft (note the small energy scale in the inset in Fig. (5).

At the transition from HM to FM state, these two points merge, resulting in the vanishing spin stiffness. Thus the transition from HM to FM state at zero temperature, which can be induced by varying $\Delta,(p d \sigma)$, or $t_{p p}$ (see Fig. 2), is a quantum critical point, at which both the Curie and Néel temperatures drop to zero due to diverging spin fluctuations. In reality such a quantum critical behavior can be suppressed by the single-ion and exchange anisotropies, neglected in our model, which open a gap in the magnon spectrum at $\mathbf{q}=\mathbf{Q}$ and result in a first order transition between the two magnetic states. This may explain, why the magnetic ordering temperature in $\mathrm{SrFeO}_{3}$ monotonously grows with pressure, even though the HM state is replaced by the FM one at around $13 \mathrm{GPa}$ [17].

In conclusion, we showed that in double exchange systems with a high density of oxygen holes the kinetic energy of the conduction electrons is minimized for a helicoidal spin ordering. This allows us to relate the helicoidal ordering observed in $\mathrm{SrFeO}_{3}$ and $\mathrm{CaFeO}_{3}$ to the negative charge transfer energy, inferred for these materials from photoemission experiments. The difference between the magnetism in ferrates and manganites is very similar to the difference between the Mott-Hubbard and charge transfer insulators 18]: the lowest energy spinflip excitation in ferrates resides mainly on oxygen sites and does not cost much energy. When the holes predomi-

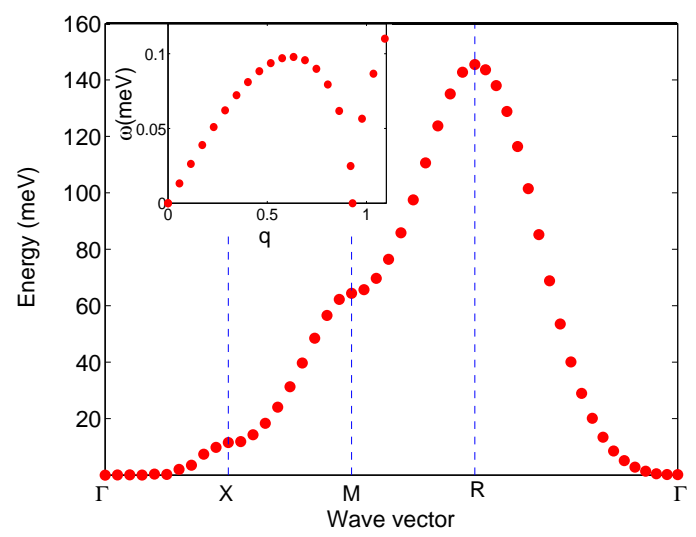

FIG. 5: The magnon spectrum for the helicoidal state with $\mathbf{Q}=0.93(1,0,0)$, which is the ground state for $(p d \sigma)=1.7 \mathrm{eV}$, $t_{p p}=0.65 \mathrm{eV}$, and $\Delta=-2 \mathrm{eV}$. The inset shows $\omega_{\mathbf{q}}$ for $\mathbf{q}=$ $q(1,0,0)$, where $q$ varies between 0 and $1.2 Q$.

nantly occupy TM sites, their kinetic energy is minimized for parallel local spins. On the other hand, the kinetic energy of the oxygen holes is minimal for the unpolarized Fermi sea. When both the transition metal and oxygen sites are occupied with high probability, the compromise is the helicoidal ordering.

I would like to thank B. Keimer, G. Khaliullin, D. Khomskii, O. Sushkov, B. Simons, and C. Ulrich for fruitful discussions.

[1] "Colossal Magnetoresistive Manganites", ed. Y. Tokura (Gordon and Breach, 2000).

[2] J.B. MacChesneyet al., J. Chem. Phys. 43, 1907 (1965).

[3] M. Takano et al., Mater. Res. Bull. 12, 923 (1977).

[4] T. Takeda, Y. Yamaguchi, and H. Watanabe, J. Phys. Soc. Jpn. 33, 967 (1972).

[5] H. Oda, Y. Yamaguchi, H. Takei, and H. Watanabe, J. Phys. Soc. Jpn. 42, 101 (1977).

[6] P.M. Woodward et al. Phys. Rev. B 62, 844 (2000).

[7] A. Lebon et al., Phys. Rev. Lett. 92, 037202 (2004).

[8] A.E. Bocquet et al., Phys. Rev. B 46, 3771 (1992).

[9] I.E. Dzyaloshinskii, Zh. Eksp. Teor. Fiz. 46, 1420 (1964) [Sov. Phys.-JETP 19, 960 (1964)].

[10] C. Zener, Phys. Rev. 81, 440 (1951); ibid 82, 403 (1951).

[11] P.W. Anderson and H. Hasegawa, Phys. Rev. 100, 675 (1955).

[12] P.-G. de Gennes, Phys. Rev. 118, 141 (1960).

[13] For a review see e.g. E. Dagotto et al., Phys. Rep. 344, 1 (2001).

[14] B. Shraiman and E.D. Siggia, Phys. Rev. Lett. 62, 1564 (1989).

[15] J. Geck et al., Phys. Rev. B, 64, 144430 (2001).

[16] F.C. Zhang and T.M. Rice, Phys. Rev. B 37, 3759 (1988).

[17] S. Nasu, T. Kawakami, S. Kawasaki, and M. Takano, Hyp. Intaract. 144/145, 119 (2002).

[18] J. Zaanen, G.A. Sawatzky, and J.W. Allen, Phys. Rev. Lett. 55, 418 (1985). J. Zaanen and G.A. Sawatzky, Can. 
J. Phys. 65, 1262 (1987). 